\title{
Evaluating current practice and outcomes of therapeutic anticoagulation during intra-aortic balloon counterpulsation in a coronary care unit
}

\author{
Jennifer L Bong BScPharm ACPR ${ }^{1}$, Ernest H Law BScPharm PharmD ACPR BCPS ${ }^{2}$, \\ Sheri L Koshman BScPharm PharmD ACPR ${ }^{3}$, Wayne J Tymchak MD FRCPC FACC ${ }^{3}$, Albert M Mogrhabi BScPharm, \\ Margaret L Ackman BScPharm PharmD ACPR FCSHP ${ }^{1}$
}

\begin{abstract}
JL Bong, EH Law, SL Koshman, WJ Tymchak, AM Mogrhabi, ML Ackman. Evaluating current practice and outcomes of therapeutic anticoagulation during intra-aortic balloon counterpulsation in a coronary care unit. Curr Res Cardiol 2015;2(1):15-18.
\end{abstract}

INTRODUCTION: Therapeutic anticoagulation for intra-aortic balloon pumps (IABPs) in coronary care unit patients is common, and a strategy of selective anticoagulation may minimize bleeding and prevent thrombosis. OBJECTIVE: The present retrospective chart review aimed to determine the proportion of patients with an IABP in place for $\geq 12 \mathrm{~h}$ during coronary care unit admission at a university-affiliated tertiary care centre from January 1, 2007 to December 31, 2011 who were eligible for selective anticoagulation, and to determine the incidence of major bleeding and ischemic complications in this population.

METHODS: Data collection was performed by one researcher using a standardized form according to prespecified definitions. Data regarding

T he intra-aortic balloon pump (IABP) is an invasive device used to support and improve hemodynamics in critically ill patients with cardiac disease $(1,2,3)$. Beneficial effects are based on the principle of 'counterpulsation', where blood is pumped or displaced out of phase with the normal cardiac cycle (1) with the overall goal of improving cardiac output. IABP is indicated in situations such as refractory unstable angina, severe ischemia, hemodynamic instability before and after a procedure (eg, coronary angiography, cardiopulmonary bypass), recurrent ischemia or infarction, complicated myocardial infarction, mechanical complications due to acute myocardial infarction or cardiogenic shock, and refractory ventricular arrhythmias $(1,4)$. Evidence supporting the use of IABP is mainly based on registry data, with several adequately powered randomized trials resulting in inconclusive evidence (2).

The use of IABP has been associated with complications including limb ischemia, systemic embolization and bleeding, as well as infection and mechanical complications due to device failure (1). Because the $\mathrm{IABP}$ is a foreign object inserted percutaneously through the femoral artery, limb ischemia secondary to embolization has been perceived as one of the most serious IABP-related complications, potentially leading to limb amputation. Therefore, anticoagulation with therapeutic heparin is often used routinely as adjunctive therapy (3).

According to a comprehensive IABP registry, the risk of limb ischemia is reported to be $2.9 \%$ (4). However, over the past 20 years, technological advances, such as the use of thin IABP catheters and patient characteristics, major bleeds, ischemic events and death were collected while the IABP was in situ and for $24 \mathrm{~h}$ after IABP removal. Descriptive statistical analysis was performed.

RESULTS: Of the 70 patients included, 50\% required an IABP for cardiogenic shock. With respect to medications, 93\% were anticoagulated and $67 \%$ received $\geq 3$ medications that could increase bleed risk. Eighty percent of patients with IABPs had at least one indication for anticoagulation while the IABP was in situ. The incidence of major bleeds and limb ischemia was $31 \%$ and $4 \%$, respectively. Twenty percent of patients died and $93 \%$ of these experienced a major bleed.

CONCLUSIONS: Most patients with IABP have an indication for anticoagulation. The incidence of limb ischemia is relatively low. Given the high incidence of major bleeding, further consideration should be given to the use of anticoagulation and risk factors for bleeding in this patient population.

Key Words: Anticoagulants; Intra-aortic balloon pump; Shock

sheathless percutaneous insertion techniques, have reduced the incidence of limb ischemia (1).

Two studies have focused on the use of anticoagulation and bleeding risks in this population by comparing strategies of universal heparin administration (all patients received intravenous therapeutic heparin) to selective heparin administration (only patients with another primary indication for systemic anticoagulation received heparin) $(3,5)$. Both studies reported very low rates of limb ischemia irrespective of anticoagulation strategy, perhaps suggesting that not all patients need to be anticoagulated $(3,5)$. If this is the case, the strategy of selective heparin may result in a reduction of major bleeding complications without increasing the risk of limb ischemia (5).

The patient population in a coronary care unit is inherently at a high risk of bleeding due to factors such as critical illness and concomitant medications (eg, antiplatelet agents). The limited evidence available regarding IABP complications supports the risk of limb ischemia being less of a concern, shifting the focus toward the risk of bleeding and use of anticoagulation. The practice of universal therapeutic anticoagulation may be more harmful than perceived. The risk of bleeding in the acute coronary syndrome (ACS) patient population is associated with significant cost, transfusions, lengthened hospitalization, and increased one-year morbidity and mortality (6). The goal of the present study was to characterize the IABP patient population, rate of complications (eg, bleeds, ischemia) and the proportion of patients who would qualify for a selective anticoagulation strategy.

${ }^{1}$ Pharmacy Services, Alberta Health Services, Edmonton, Alberta; ${ }^{2}$ Department of Pharmacy Systems, Outcomes and Policy, University of Illinois at

Chicago, Chicago, Illinois, USA; ${ }^{3}$ Division of Cardiology, Faculty of Medicine and Dentistry, University of Alberta, Edmonton, Alberta

Correspondence: Mr Ermest Law, Department of Pharmacy Systems, Outcomes and Policy, 833 South Wood Street, MC 871, Chicago, Illinois 60612-7231,

USA. Telephone 312-996-0612, fax 312-996-2954, e-mail elaw3@uic.edu 
TABLE 1

Patient characteristics $(n=70)$

\begin{tabular}{lc}
\hline Mean age, years & 63 \\
Female sex & $17(24.3)$ \\
Comorbidities & \\
Hypertension & $34(48.6)$ \\
Dyslipidemia & $30(42.9)$ \\
Tobacco use & $24(34.3)$ \\
Diabetes & $19(27.1)$ \\
Previous coronary artery disease & $16(22.8)$ \\
Chronic kidney disease & $6(8.6)$ \\
Medications before admission & \\
Antiplatelet & $25(35.7)$ \\
Warfarin & $4(5.7)$ \\
Nonsteroidal anti-inflammatory drug & $2(2.9)$ \\
\hline Data presented as $n(\%)$ unless otherwise specified
\end{tabular}

Data presented as $n$ (\%) unless otherwise specified

The primary objective was to determine the proportion of therapeutically anticoagulated patients with an IABP who have $\geq 1$ indication for anticoagulation. The secondary objectives were to determine the incidence of major bleeding and ischemic complications in patients with IABP.

\section{METHODS}

\section{Study design and data sources}

The present retrospective descriptive study included all patients with an IABP in place for $\geq 12 \mathrm{~h}$ during their admission to a coronary care unit (University of Alberta Hospital and the Mazankowski Alberta Heart Institute, Edmonton, Alberta) from January 1, 2007 to December 31, 2011. For patients with multiple admissions, only the first hospital admission fulfilling the inclusion criteria was included. Exclusion criteria included patients with an IABP in place for $<12 \mathrm{~h}$ before transfer to the operating room or patients who required an IABP $<12 \mathrm{~h}$.

Data were abstracted from medical records by a single researcher (JB) using a standardized data collection form according to prespecified standard definitions. Data included patient characteristics (age, sex, comorbidities, medical condition[s] requiring anticoagulation, medications taken before admission), IABP information (indication, sheath or sheathless, duration of insertion, medications received while in situ) and outcomes (bleeding, ischemia or death occurring while IABP in situ and $24 \mathrm{~h}$ post-IABP removal).

\section{Study definitions}

Indications for selective anticoagulation included: unrevascularized ACS (unstable angina, non-ST-segment elevation myocardial infarction, and ST-segment elevation myocardial infarction; duration of anticoagulation therapy: $48 \mathrm{~h}$, as per the American College Cardiology/American Heart Association guidelines), awaiting percutaneous coronary intervention/coronary artery bypass graft, large anterior myocardial infarction with an ejection fraction $\leq 35 \%$ as documented in echocardiogram report, intracardiac thrombus documented in patient's chart or indicated in echocardiogram (transesophageal echocardiogram/transthoracic echocardiogram), atrial fibrillation/ flutter, artificial heart valve (mechanical or bioprosthetic within three months), recent or current venous thromboembolism (deep vein thrombosis or pulmonary embolism; duration of anticoagulation depending on risk factors, etiology, hypercoaguable state) (7), and other indication if patient on anticoagulation upon admission.

Major bleeding (according to International Society on Thrombosis and Haemostasis [ISTH] criteria) had to fulfill one of the following criteria: fatal bleeding or symptomatic bleeding in a critical area or organ (eg, intracranial, intraspinal, intraocular, retroperitoneal, intraarticular, pericardial or intramuscular), a hemoglobin decrease of $>20 \mathrm{~g} / \mathrm{L}$ or a bleed requiring $>2$ units transfusion (8).
Major limb ischemia was defined as documented in patient's chart (progress notes, nursing notes) with terms including "limb ischemia", "limb thrombus", "limb emboli" and "clot in lower limb".

\section{Statistical analysis}

Data were analyzed descriptively. The primary end point was the proportion of therapeutically anticoagulated patients with an IABP with $\geq 1$ indication for anticoagulation. Secondary end points were the percentage of patients with major bleeds or limb ischemia while the IABP was in place and $24 \mathrm{~h}$ after IABP removal, reported for the entire patient group and separately for patients with an indication for anticoagulation and those without.

\section{RESULTS}

A total of 115 charts were reviewed and 70 patients were included in the analysis. Of the 45 patients excluded, 21 patients had an IABP in place for $<12 \mathrm{~h}$ and $24 \mathrm{had}$ an IABP in place for $<12 \mathrm{~h}$ before transfer to the operating room. Characteristics of the patient population are presented in Table 1 . The mean age was 63 years and approximately one-quarter of the patients were women. Hypertension and dyslipidemia were the most common comorbidities. Approximately onethird of the patients were taking acetylsalicylic acid before admission.

IABP characteristics are presented in Table 2. The most common indication for IABP use was cardiogenic shock (47.1\%), followed by refractory ischemia $(24.3 \%)$ and hemodynamic instability before a procedure $(22.9 \%)$. Ninety-three percent of patients were anticoagulated during IABP therapy and the majority of these patients received intravenous unfractionated heparin. Sixty-seven percent of patients received $\geq 3$ medications that could increase the risk of bleeding.

Eighty percent (56 of 70) of patients had an indication for anticoagulation for the duration of balloon pump placement. Indications for anticoagulation (Table 3), in descending frequency, were ACS $(64.3 \%)$, awaiting procedure $(30.0 \%)$, atrial fibrillation/flutter $(11.4 \%)$, large anterior myocardial infarction $(7.1 \%)$ and artificial heart valve $(1.4 \%)$, with $47.1 \%$ of patients having $>1$ indication for anticoagulation. Fourteen patients $(20 \%)$ did not have an indication for selective anticoagulation while the IABP was in place.

Three $(4.3 \%)$ patients developed limb ischemia with the IABP in situ. All three of these patients were anticoagulated. One occurred in a patient who had an indication for anticoagulation and two occurred in patients who did not have an indication for anticoagulation.

The incidence of ISTH-defined major bleeding was 31\%, with 22 documented major bleeds (Table 4). Twenty of the major bleeds occurred in patients who were anticoagulated. Sixteen major bleeds occurred in patients who had an indication for anticoagulation, and six occurred in patients who did not have an indication for anticoagulation. One of the major bleeds was an access site bleed. Fourteen patients (20\%) died, eight of whom also experienced a major bleed.

\section{DISCUSSION}

In the present study, the majority of patients had an indication for anticoagulation while the IABP was in place. Limb ischemia, which has often been regarded as one of the most catastrophic complications of IABP use, was found to be $4.3 \%$, which compares favourably with the incidence reported in IABP registries $(2.9 \%)$. Similarly, the incidence of access site bleeds were also low, at $1.4 \%$, similar to $2.4 \%$ reported in the same registries (4). This may have been due to changes in IABP technology (eg, use of thinner and less thrombogenic catheters), techniques (eg, sheathless procedures) and/or improvement in the selection of patients suitable for the IABP insertion.

Conversely, the rate of major bleeding in our study, as defined by ISTH, were higher than those reported in contemporary IABP studies $(31.4 \%$ versus $11.8 \%$ to $14.1 \%)(3,5)$. It is possible that these discordances are explained by the different definitions of major bleeding chosen. For example, some studies considered major bleeding as those occurring only at the site of IABP insertion (4) and these rates were observed to be lower ( $0.8 \%$ to $1 \%)$. If we apply these definitions to our 
TABLE 2

Intra-aortic balloon pump (IABP) characteristics and medications

\begin{tabular}{lc}
\hline & $\mathbf{n}(\%)$ \\
\hline Indication for IABP & \\
Cardiogenic shock & $33(47.1)$ \\
Myocardial ischemia & $17(24.3)$ \\
Hemodynamically unstable before procedure & $16(22.9)$ \\
Myocardial infarction & $7(10.0)$ \\
$\quad$ Mechanical & $1(14.3)$ \\
Chronic heart failure/left ventricular failure & $6(85.7)$ \\
Other & $4(5.7)$ \\
Hemodynamically unstable after procedure & $2(2.9)$ \\
Arrhythmias & $1(1.4)$ \\
IABP & \\
Sheath & $46(65.7)$ \\
Sheathless & $23(32.9)$ \\
Unknown & $1(1.4)$ \\
Duration of IABP in situ, h, mean (range) & $75(12-309.5)$ \\
Medications received while IABP in situ & \\
Anticoagulation & $65(92.9)$ \\
Intravenous unfractionated heparin & $63(90)$ \\
Low molecular weight heparin ${ }^{\dagger}$ & $12(17.1)$ \\
Warfarin & $1(1.4)$ \\
Other & $1(1.4)$ \\
Medications that could increase bleed risk & $65(92.9)$ \\
Unfractionated heparin (sc for DVT prophylaxis) & $10(14.3)$ \\
Low molecular weight heparin (sc for DVT prophylaxis) & $1(1.4)$ \\
Glycoprotein 2a3b inhibitor & $24(34.3)$ \\
Abciximab & $15(62.5)$ \\
Eptifibatide & $9(37.5)$ \\
Low-dose acetylsalicylic acid & $60(85.7)$ \\
Clopidogrel & $45(64.3)$ \\
Fibrinolytics & $4(5.7)$ \\
Other & $3(4.3)$ \\
Total number of medications received that could & \\
$<3$ & $23(32.9)$ \\
$\geq 3$ & $47(67.1)$ \\
\hline
\end{tabular}

*Documented as: "ventricular tachycardic arrest in cardiac catheterization laboratory, bradycardia and hypotension"; ${ }^{\dagger}$ Patients could be switched from intravenous unfractionated heparin to low molecular weight heparin or vice versa; ${ }^{\ddagger}$ Lepirudin $(n=1) ;{ }^{\S}$ Bivalirudin $(n=1)$, dipyrimadole $(n=1)$ and prasugrel $(n=1)$. DVT Deep vein thrombosis; sc Subcutaneous

study, we had a similar rate of one (1.4\%) major access site bleed. In contrast, other studies that used a broader definition that included any bleeding associated with hemodynamic compromise requiring blood transfusion or surgical intervention with or without intracranial hemorrhage, observed higher rates of $10.8 \%$ to $14.1 \%(3,5)$. Our study found no documentation of intracranial hemorrhage and it is not known whether the 17 patients (24\%) who received transfusions did so due to hemodynamic compromise because this information was not collected in our study. Eight deaths occurred in patients who also experienced a major bleed. Because we were unable to attribute cause of death, we were unable to compare our rates with other studies that used these definitions. We were interested in assessment of the risk associated with anticoagulation for IABPs; thus, we chose to define major bleeding based on definitions outlined by ISTH, which has been used in cardiology clinical trials of similar patient populations (8). The ISTH criteria uses more conservative laboratory measurements and broader clinical assessment - overall, a clinically relevant definition of major bleeding.
TABLE 3

Indications for selective anticoagulation

\begin{tabular}{lc}
\hline & $\mathbf{n}(\%)$ \\
\hline Atrial fibrillation/flutter & $8(11.4)$ \\
Artificial heart valve & $1(1.4)$ \\
Mechanical & $1(1.4)$ \\
Bioprosthetic & 0 \\
Acute coronary syndrome & $45(64.3)$ \\
Unstable angina & $3(6.7)$ \\
NSTEMI & $17(37.8)$ \\
STEMI & $25(55.6)$ \\
Awaiting procedure & $21(30.0$ \\
Percutaneous coronary intervention & 0 \\
Coronary artery bypass graft & $21(30.0)$ \\
Large anterior myocardial infarction & $5(7.1)$ \\
*Some patients had >1 indication for selective anticoagulation. NSTEMI Non- \\
ST-segment elevation myocardial infarction; STEMI ST-segment elevation \\
myocardial infarction
\end{tabular}

\section{TABLE 4}

Characterization of bleeding

\begin{tabular}{lc} 
Criteria & $\mathbf{n}(\%)$ \\
\hline Fatal bleeding* & $8(11.4)$ \\
Intracranial bleeding & $0(0)$ \\
Hemoglobin decrease of $>20 \mathrm{~g} / \mathrm{L}$ & $14(20.0)$ \\
Blood transfusion (any associated with bleeding) & $17(24.3)$ \\
Blood transfusion $>2$ units & $8(11.4)$ \\
Endoscopic examination & $3(4.3)$ \\
ISTH (fatal bleeding or symptomatic bleeding in a critical & $22(31.4)$ \\
$\quad$ area or organ, hemoglobin decrease $>20 \mathrm{~g} / \mathrm{L}$, blood & \\
transfusion $>2$ units) & \\
\hline$n=70 .{ }^{*}$ Defined as death in patient who experienced a major bleed. ISTH \\
International Society on Thrombosis and Haemostasis
\end{tabular}

The 20\% mortality rate is similar to rates reported in previous studies $(3,4)$, which reflects the high acuity of illness in this patient population. The high incidence of a major bleed events among patients who died is consistent with the poor prognosis associated with major bleeding in ACS patients (6). This further emphasizes the importance of characterizing the bleed risk in these patients.

The retrospective descriptive study design allowed for five years of data of a patient cohort to be analyzed, as well as the ability to characterize relatively rare occurrences (ie, limb ischemia). However, there are inherent limitations to retrospective descriptive studies. Foremost is that cause-and-effect relationships cannot be established because many confounders exist within the patient population itself. With respect to identifying an indication for anticoagulation, classification was performed retrospectively based on prespecified definitions. In practice, the clinical decision for indications requiring ongoing anticoagulation is not always as unequivocal. Additionally, we were limited in our ability to characterize the ongoing indication for anticoagulation in each patient (eg, the duration of anticoagulation for an ACS patient). Data were collected from medical records; however strategies were implemented to standardize the data collection process.

The present study provides a current report of IABP practice with respect to anticoagulation, patient population and its associated risks. Although the majority of patients had an indication for anticoagulation while the IABP was in place, the present study suggests that, given the high incidence of major bleeding, further consideration and concern should be placed on the use of anticoagulation (ie, consideration of selective heparin strategy) and risk factors for bleeding in this patient population. It may also be appropriate to investigate other patient populations in whom the IABP technology is used (eg, cardiovascular surgery). 


\section{CONCLUSIONS}

In the patient population studied, the majority of patients have an indication for anticoagulation while the IABP is in place. A higher incidence of major bleeding was observed while the risk of limb ischemia is reassuringly low. Further consideration should be given to the use of anticoagulation and risk factors for bleeding in this patient population.

ACKNOWLEDGEMENTS: Alberta Pharmacy Practice Residency Program.

\section{REFERENCES}

1. Cohen MG, Ohman EM, Santa-Cruz RA. Aortic counterpulsation: A review of the hemodynamic effects and indications for use. Catheter Cardiovasc Interv 2006;67:68-77.

2. Thiele H, Zeymer U, Neumann FJ, et al. Intraaortic balloon support for myocardial infarction with cardiogenic shock. N Engl J Med 2012;367:1287-96.

3. Jiang CY, Zhao LL, Wang JA, Mohammod B. Anticoagulation therapy in intra-aortic balloon counterpulsation: Does IABP really need anti-coagulation? J Zhejiang Univ Sci 2003;4:607-11.

4. Ferguson JJ 3rd, Cohen M, Freedman RJ Jr, et al. The current practice of intra-aortic balloon counterpulsation: Results from the benchmark registry. J Am Coll Cardiol 2001;38:1456-62

5. Cooper HA, Thompson E, Panza JA. The role of heparin anticoagulation during intra-aortic balloon counterpulsation in the coronary care unit. Acute Cardiac Care 2008;10:214-220.

\section{SOURCES OF FUNDING: None.}

DISCLOSURES: Dr Ernest Law was supported by a 2014-2016 UIC/ Takeda Fellowship in Health Economics and Outcomes Research at the time of revisions. Dr Wayne Tymchak has received honoraria from AstraZeneca for providing lectures and participating on an advisory board.

6. Dauerman HL, Rao SV, Resnic FS, et al. Bleeding avoidance strategy consensus and controversy. J Am Coll Cardiol 2011;58:1-10.

7. Hirsch J, Hoak J. Management of deep vein thrombosis and pulmonary embolism: A statement for health care professionals from the council on thrombosis (in consultation with the council on cardiovascular radiology), American Heart Association. Circulation 1996;93:2212-45.

8. Steg PG, Huber K, Andreotti F, et al. Bleeding in acute coronary syndromes and percutaneous coronary interventions: Position paper by the Working Group on Thrombosis of the European Society of Cardiology. Eur Heart J 2011;32:1854-64. 